\title{
ELECTRICAL OSCILLATIONS IN ANTENNAS AND INDUCTANCE COILS
}

\author{
By John M. Miller
}

\section{CONTENTS}

I.

I. Introduction $\ldots \ldots \ldots \ldots \ldots \ldots \ldots \ldots \ldots \ldots \ldots \ldots \ldots \ldots \ldots \ldots \ldots$

II. Circuit with uniformly distributed inductance and capacity ........679

III. The Antenna . . . . . . . . . . . . . . . . . . . . . . . . . 680

I. Reactance of the aerial-ground portion $\ldots \ldots \ldots \ldots \ldots \ldots \ldots \ldots \ldots$

2. Natural frequencies of oscillation $\ldots \ldots \ldots \ldots \ldots \ldots \ldots \ldots \ldots \ldots$ I

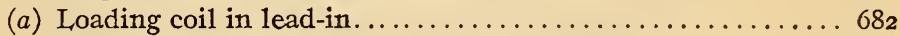

(b) Condenser in lead-in. ........................ $68_{4}$

3. Effective resistance, inductance, and capacity .............. $68_{5}$

4. Equivalent circuit with lumped constants.................. 689

5. Determination of static capacity and inductance..............690

6. Determination of effective resistance, inductance, and capacity ... 692

IV. The Inductance coil . . . . . . . . . . . . . . . . . . . . . . . . . 694

I. Reactance of the coil . . . . . . . . . . . . . . . . . . . . . 694

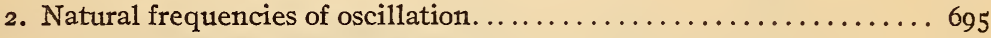

Condenser across the terminals.....................6 695

3. Equivalent circuit with lumped constants................. 695

\section{INTRODUCTION}

A modern radiotelegraphic antenna generally consists of two portions, a vertical portion or "lead-in" and a horizontal portion or "aerial." At the lower end of the lead-in, coils or condensers or both are inserted to modify the natural frequency of the electrical oscillations in the system. When oscillating, the current throughout the entire lead-in is nearly constant and the inductances, capacities, and resistances in this portion may be considered as localized or lumped. In the horizontal portion, however, both the strength of the current and the voltage to earth vary from point to point and the distribution of current and voltage varies with the frequency. The inductance, capacity, and resistance of this portion must therefore be considered as distributed throughout its extent and its effective inductance, capacity, and resistance will depend upon the frequency. On this account the mathematical treatment of the oscillations of an antenna is not $110990^{\circ}-19-13$ 
as simple as that which applies to ordinary circuits in which all of the inductances and capacities may be considered as lumped.

The theory of circuits having uniformly distributed electrical characteristics such as cables, telephone lines, and transmission lines has been applied to antennas. The results of this theory do not seem to have been clearly brought out; hazy and sometimes erroneous ideas appear to be current in the literature, textbooks, and in the radio world in general so that the methods of antenna measurements are on a dubious footing. It is hoped that this paper may clear up some of these points. No attempt has been made to show how accurately this theory applies to actual antennas.

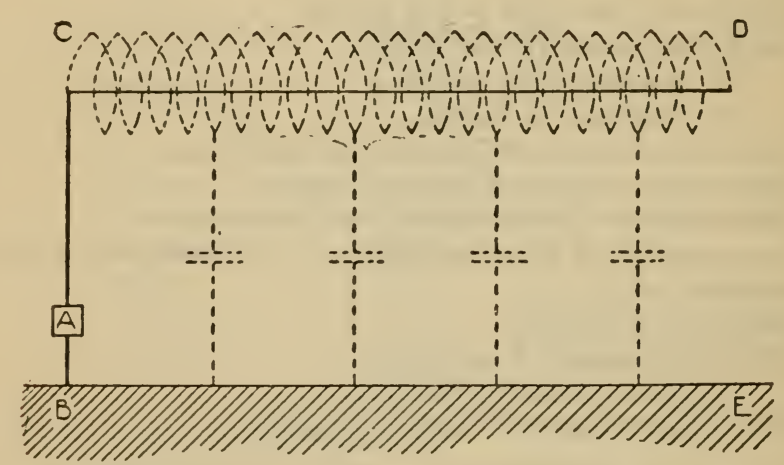

FIG. I.-Antenna represented as a line with uniform distribution of inductance and capacity

The aerial-ground portion of the antenna, or aerial for short ( $C D$ in Fig. I), will be treated as a line with uniformly distributed inductance, capacity, and resistance. As is common in the treatment of radio circuits the resistance will be considered to be so low as not to affect the frequency of the oscillations or the distribution of current and voltage. The lead-in, $B C$ in Fig. I, will be considered to be free from inductance or capacity excepting as inductance coils or condensers are inserted at $A$ to modify the oscillations.

An inductance coil, particularly if a long single-layer solenoid, may also be treated from the standpoint of the transmission-line theory. The theoretical results obtained furnish an interesting explanation of certain well-known experimental results. 


\section{CIRCUIT WITH UNIFORMLY DISTRIBUTED INDUCTANCE AND CAPACITY}

The theory, generally applicable to all circuits with uniformly distributed inductance and capacity, will be developed for the case of two parallel wires. The wires (Fig. 2) are of length $l$ and of low resistance. The inductance per unit length $L_{1}$ is defined by the flux of magnetic force between the wires per unit of length that there would be if a steady current of I ampere were flowing in opposite directions in the two wires. The capacity per unit length $C_{1}$ is defined by the charge that there would be on a unit length of one of the wires if a constant emf of I volt were impressed between the wires? Further, the quantity $L_{0}=l L_{1}$ would be the total inductance of the circuit if the current flow were the same at all parts. This would be the case if a constant or slowly

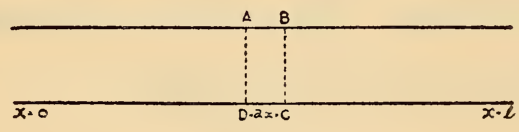

FIG. 2

alternating voltage were applied at $x=0$ and the far end $(x=l)$ short-circuited. The quantity $C_{0}=l C_{1}$ would represent the total capacity between the wires if a constant or slowly alternating voltage were applied at $x=0$ and the far end were open.

Let it be assumed, without defining the condition of the circuit at $x=l$, that a sinusoidal emf of periodicity $\omega=2 \pi f$ is impressed at $x=0$ giving rise to a current of instantaneous value $i$ at $A$ and a voltage between $A$ and $D$ equal to $v$. At $B$ the current will be $i+\frac{\delta i}{\delta x} d x$ and the voltage from $B$ to $C$ will be $v+\frac{\delta v}{\delta x} d x$.

The voltage around the rectangle $A B C D$ will be equal to the rate of decrease of the induction through the rectangle, hence

$$
\begin{gathered}
\left(v+\frac{\delta v}{\delta x} d x\right)-v=-\frac{\delta}{\delta t}\left(L_{1} i d x\right) \\
\frac{\delta v}{\delta x}=-L_{1} \frac{\delta i}{\delta t}
\end{gathered}
$$

Further, the rate of increase of the charge $q$ on the elementary length of wire $A B$ will be equal to the excess in the current flowing in at $A$ over that flowing out at $B$. 
Hence

$$
\begin{aligned}
\frac{\delta q}{\delta t}=\frac{d}{d t}\left(C_{1} v d x\right) & =i-\left(i+\frac{\delta i}{\delta x} d x\right) \\
-\frac{\delta i}{\delta x} & =C_{1} \frac{\delta v}{\delta t}
\end{aligned}
$$

These equations (I) and (2) determine the propagation of the current and voltage waves along the wires. In the case of sinusoidal waves, the expressions

$$
\begin{aligned}
& v=\cos \omega t\left(A \cos \omega \sqrt{C_{1} L_{1}} x+B \sin \omega \sqrt{C_{1} L_{1}} x\right) \\
& i=\sin \omega t \sqrt{\frac{C_{1}}{L_{1}}}\left(A \sin \omega \sqrt{C_{1} L_{1}} x-B \cos \omega \sqrt{C_{1} L_{1}} x\right)
\end{aligned}
$$

are solutions of the above equations as may be verified by substitution. The quantities $A$ and $B$ are constants depending upon the terminal conditions. The velocity of propagation of the waves, at high frequencies is $V=\frac{I}{\sqrt{C_{1} L_{1}}}$.

\section{THE ANTENNA}

\section{REACTANCE OF THE AERIAL-GROUND PORTION}

Applying equations (3) and (4) to the aerial of an antenna and assuming that $x=0$ is the lead-in end while $x=l$ is the far end which is open, we may introduce the condition that the current is zero for $x=l$. From (4)

$$
\frac{A}{B}=\cot \omega \sqrt{C_{1} L_{1}} l
$$

Now the reactance of the aerial, which includes all of the antenna but the lead-in, is given by the current and voltage at $x=0$. These are, from (3), (4), and (5),

$$
\begin{aligned}
& v_{0}=A \cos \omega t=B \cot \omega \sqrt{C_{1} L_{1}} l \cos \omega t \\
& i_{0}=-\sqrt{\frac{C_{1}}{L_{1}}} B \sin \omega t .
\end{aligned}
$$

The current leads the voltage when the cotangent is positive and lags when the cotangent is negative. The reactance of the aerial, given by the ratio of the maximum values of $v_{0}$ to $i_{o}$, is

$$
X=-\sqrt{\frac{L_{1}}{C_{1}}} \cot \omega \sqrt{C_{1} L_{1} l}
$$


or in terms of $C_{0}=l C_{1}$ and $L_{0}=l L_{1}$

$$
X=-\sqrt{\frac{L_{0}}{C_{0}}} \cot \omega \sqrt{C_{0} L_{0}}
$$

or since

$$
V=\frac{\mathrm{I}}{\sqrt{L_{1} C_{1}}}
$$

$X=-L_{1} V \cot \omega \sqrt{C_{1} L_{1}} l$ as given by J. S. Stone. ${ }^{1}$

At low frequencies the reactance is negative and hence the aerial behaves as a capacity. At the frequency $f=\frac{I}{4 \sqrt{C_{0} L_{0}}}$

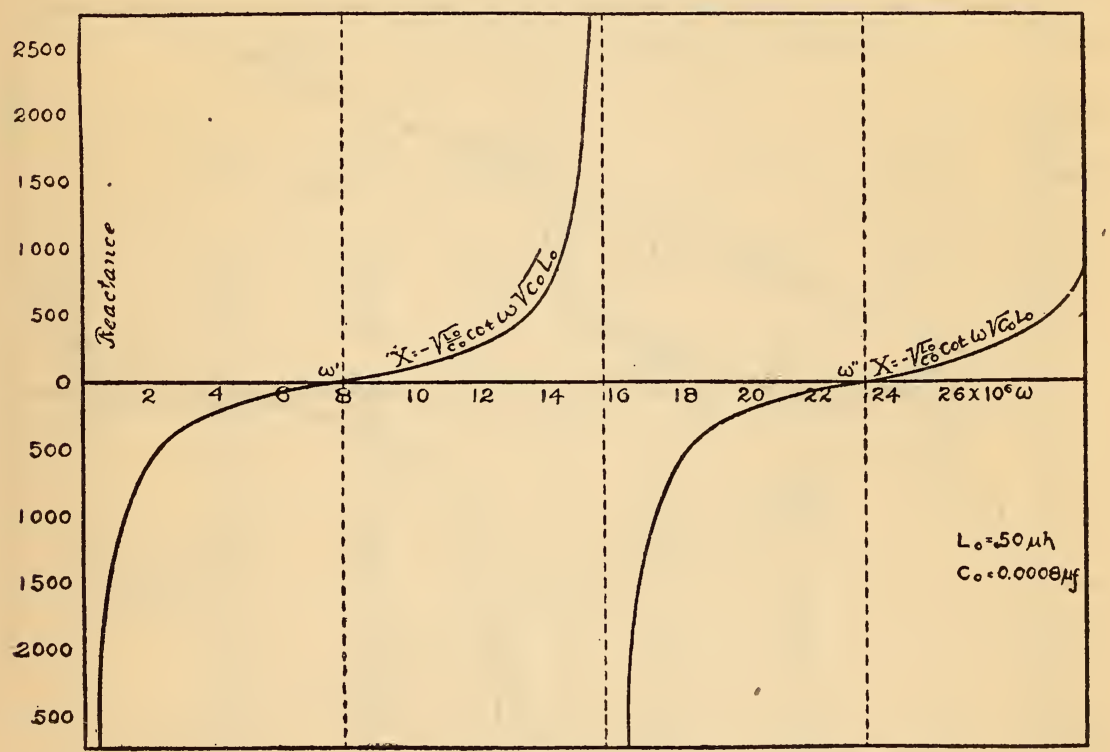

FIG. 3.-Variation of the reactance of the aerial of an antenna with the frequency

the reactance becomes zero and beyond this frequency is positive or inductive up to the frequency $f=\frac{\mathrm{I}}{2 \sqrt{C_{0} L_{0}}}$ at which the reactance becomes infinite. This variation of the aerial reactance with the frequency is shown by the cotangent curves in Fig. 3 .

\section{NATURAL FREQUENCIES OF OSCILLATION}

Those frequencies at which the reactance of the aerial, as given by equation (6), becomes equal to zero are the natural frequencies of oscillation of the antenna (or frequencies of resonance) when the 
lead-in is of zero reactance. They are given in Fig. 3 by the points of intersection of the cotangent curves with the axis of ordinates and by the equation

$$
f=\frac{m}{4 \sqrt{C_{0} L_{0}}} ; m=\mathrm{I}, 3,5 \text {, etc. }
$$

The corresponding wave lengths are given by

$$
\lambda=\frac{V}{f}=\frac{l}{f \sqrt{C_{0} L_{0}}}=\frac{t l}{m}
$$

that is, $4 / \mathrm{I}, 4 / 3,4 / 5,4 / 7$, etc., times the length of the aerial. If, however, the lead-in has a reactance $X_{X}$, the natural frequencies

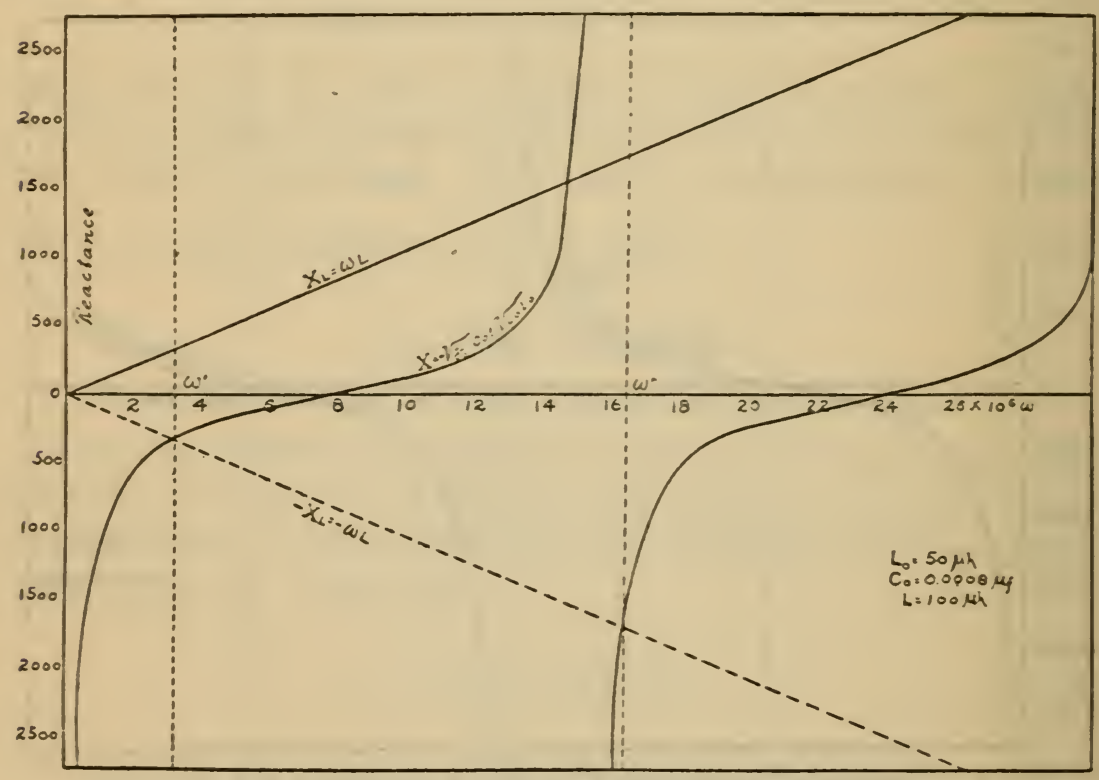

FIG. 4.-Curves of aerial and loading coil reactances

of oscillation are determined by the condition that the total reactance of lead-in plus aerial shall be zero; that is,

$$
X_{\mathrm{X}}+X=0
$$

provided that the reactances are in series with the driving emf.

(a) Loading Coil in Lead-in. - The most important practical case is that in which an inductance coil is inserted in the lead-in. If the coil has an inductance $L$, its reactance $X_{L}=\omega L$. This is a positive reactance increasing linearly with the frequency and represented in Fig. 4 by a solid line. Those frequencies at which the reactance of the coil is equal numerically but opposite in sign 
to the reactance of the aerial, are the natural frequencies of oscillation of the loaded antenna since the total reactance $X_{L}+X=0$. Graphically, these frequencies are determined by the intersection of the straight line $-X_{L}=-\omega L$ (shown by a dash line in Fig. 4) with the cotangent curves representing $X$. It is evident that the frequency is lowered by the insertion of the loading coil and that the higher natural frequencies of oscillation are no longer integral multiples of the lowest frequency.

The condition $X_{L}+X=0$, which determines the natural frequencies of oscillation, leads to the equation

$$
\omega L-\sqrt{\frac{L_{0}}{C_{0}}} \cot \omega \sqrt{C_{0} L_{0}}=0 .
$$

TABLE 1.-Data for Loaded Antenna Calculations

\begin{tabular}{|c|c|c|c|c|c|c|c|}
\hline$\frac{\mathrm{L}}{\mathrm{L}_{0}}$ & $\omega \sqrt{\mathrm{C}_{0} \mathrm{I}_{\mathrm{o}}}$ & $\frac{1}{\sqrt{\frac{\bar{L}}{\bar{L}_{0}}+\frac{1}{3}}}$ & $\begin{array}{c}\text { Differ- } \\
\text { ence, per } \\
\text { cent }\end{array}$ & $\frac{L}{L_{0}}$ & $\omega \sqrt{\mathrm{C}_{0} \mathrm{I}_{\mathrm{o}}}$ & $\frac{1}{\sqrt{\frac{L}{L_{0}}+\frac{1}{3}}}$ & $\begin{array}{c}\text { Differ- } \\
\text { ence, per } \\
\text { cent }\end{array}$ \\
\hline $\begin{array}{l}0.0 \\
.1 \\
.2 \\
.3 \\
.4\end{array}$ & $\begin{array}{l}1.571 \\
1.429 \\
1.314 \\
1.220 \\
1.142\end{array}$ & $\begin{array}{l}1.732 \\
1.519 \\
1.369 \\
1.257 \\
1.168\end{array}$ & $\begin{array}{r}10.3 \\
6.3 \\
4.2 \\
3.0 \\
2.3\end{array}$ & $\begin{array}{l}3.2 \\
3.3 \\
3.4 \\
3.5\end{array}$ & $\begin{array}{r}0.532 \\
.524 \\
.517 \\
.510\end{array}$ & $\begin{array}{r}0.532 \\
.525 \\
.518 \\
.511\end{array}$ & $\begin{array}{l}0.1 \\
.1 \\
.1 \\
.1\end{array}$ \\
\hline $\begin{array}{l}.5 \\
.6 \\
.7 \\
.8 \\
.9\end{array}$ & $\begin{array}{r}1.077 \\
1.021 \\
.973 \\
.931 \\
.894\end{array}$ & $\begin{array}{r}1.095 \\
1.035 \\
.984 \\
.939 \\
.900\end{array}$ & $\begin{array}{r}1.7 \\
1.4 \\
1.1 \\
.9 \\
.7\end{array}$ & $\begin{array}{l}3.6 \\
3.7 \\
3.8 \\
3.9 \\
4.0\end{array}$ & $\begin{array}{l}.304 \\
.4977 \\
.4916 \\
.4859 \\
.4801\end{array}$ & $\begin{array}{l}.504 \\
.4979 \\
.4919 \\
.4860 \\
.4804\end{array}$ & $\begin{array}{l}.0 \\
.0 \\
.0 \\
.0 \\
.0\end{array}$ \\
\hline $\begin{array}{l}1.0 \\
1.1 \\
1.2 \\
1.3 \\
1.4\end{array}$ & $\begin{array}{l}.860 \\
.831 \\
.804 \\
.779 \\
.757\end{array}$ & $\begin{array}{l}.866 \\
.835 \\
.808 \\
.782 \\
.760\end{array}$ & $\begin{array}{l}.7 \\
.5 \\
.5 \\
.4 \\
4\end{array}$ & $\begin{array}{l}4.5 \\
5.0 \\
5.5 \\
6.0 \\
6.5\end{array}$ & $\begin{array}{r}.4548 \\
.4330 \\
.4141 \\
.3974\end{array}$ & $\begin{array}{r}.4549 \\
.4330\end{array}$ & .00 \\
\hline $\begin{array}{l}1.5 \\
1.6 \\
1.7 \\
1.8 \\
1.9\end{array}$ & $\begin{array}{l}.735 \\
.717 \\
.699 \\
.683 \\
.668\end{array}$ & $\begin{array}{l}.739 \\
.719 \\
.701 \\
.685 \\
.669\end{array}$ & $\begin{array}{l}.4 \\
.3 \\
.3 \\
.3 \\
.3\end{array}$ & $\begin{array}{l}7.0 \\
7.5 \\
8.0 \\
8.5 \\
9.0\end{array}$ & $\begin{array}{r}.38690 \\
.3574 \\
.3465 \\
.3366 \\
.3275\end{array}$ & & \\
\hline $\begin{array}{l}2.0 \\
2.1 \\
2.2 \\
2.3 \\
2.4\end{array}$ & $\begin{array}{l}.653 \\
.640 \\
.627 \\
.615 \\
.604\end{array}$ & $\begin{array}{l}.655 \\
.641 \\
.628 \\
.6016\end{array}$ & $\begin{array}{l}.3 \\
.2 \\
.2 \\
.2 \\
.2\end{array}$ & $\begin{array}{r}9.5 \\
1.0 \\
11.0 \\
12.0 \\
13.0\end{array}$ & $\begin{array}{r}.3189 \\
.3111 \\
.2972 \\
.2850 \\
.2741\end{array}$ & & \\
\hline $\begin{array}{l}2.5 \\
2.6 \\
2.7 \\
2.8 \\
2.9\end{array}$ & $\begin{array}{l}.593 \\
.583 \\
.574 \\
.564 \\
.556\end{array}$ & $\begin{array}{l}.594 \\
.584 \\
.574 \\
.565 \\
.556\end{array}$ & $\begin{array}{l}.2 \\
.2 \\
.2 \\
.1\end{array}$ & $\begin{array}{l}14.0 \\
15.0 \\
16.0 \\
17.0 \\
18.0\end{array}$ & $\begin{array}{l}.2644 \\
.2556 \\
.2476 \\
.2402 \\
.2338\end{array}$ & & \\
\hline $\begin{array}{l}3.0 \\
3.1\end{array}$ & $\begin{array}{r}.547 \\
.539\end{array}$ & $\begin{array}{r}.548 \\
.540\end{array}$ & $: 1$ & $\begin{array}{l}19.0 \\
20.0\end{array}$ & $\begin{array}{l}.22277 \\
.2219\end{array}$ & & \\
\hline
\end{tabular}

or

$$
\frac{\cot \omega \sqrt{C_{\mathrm{o}} L_{\mathrm{o}}}}{\omega \sqrt{C_{\mathrm{o}} L_{\mathrm{o}}}}=\frac{L}{L_{\mathrm{o}}}
$$

This equation has been given by Guyau ${ }^{2}$ and L. Cohen. ${ }^{3}$ It

${ }^{2}$ A. Guyau, Lumiere Electrique, 15, p. r3ł rgir. ${ }^{3}$ L. Cohen, Electrical World, 65, p. 286; I9I5. 
determines the periodicity $\omega$ and hence the frequency and wave length of the possible natural modes of oscillation when the distributed capacity and inductance of the aerial and the inductance of the loading coil are known. This equation can not, however, be solved directly; it may be solved graphically, as shown in Fig. 4, or a table may be prepared indirectly which gives the values of $\omega \sqrt{C_{0} L_{0}}$ for different values of $\frac{L}{L_{0}}$, from which then $\omega, f$, or $\lambda$ may be determined. The second column of Table I gives these values for the lowest natural frequency of oscillation, which is of the major importance practically.

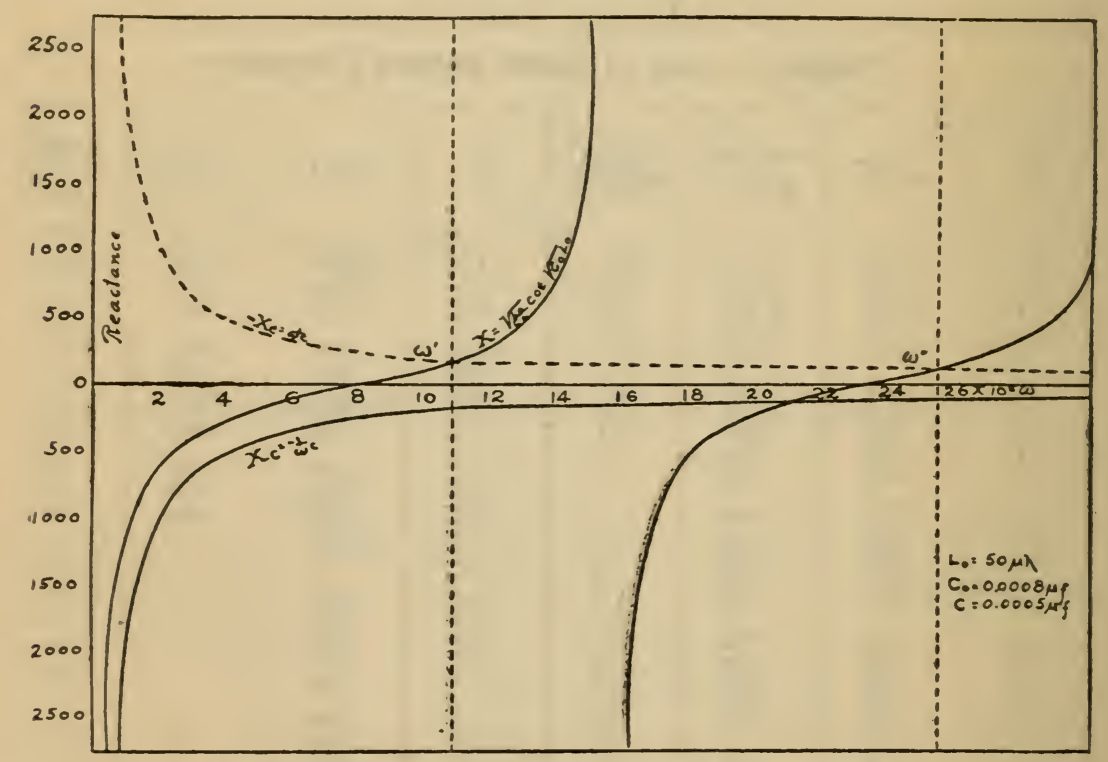

FIG. 5.-Curves of aerial and series condenser reactances

(b) Condenser in Lead-in.-At times in practice a condenser is inserted in the lead-in. If the capacity of the condenser is $C$, its reactance is $X_{0}=-\frac{\mathrm{I}}{\omega C}$. This reactance is shown in Fig. 5 by the hyperbola drawn in solid line. The intersection of the negative of this curve (drawn in dash line) with the cotangent curves representing $X$ gives the frequencies for which $X_{c}+X=0$, and hence the natural frequencies of oscillation of the antenna. The frequencies are increased (the wave length decreased) by the insertion of the condenser and the oscillations of higher frequencies are not integral multiples of the lowest. 
The condition $X_{\mathrm{c}}+X=o$ is expressed by the equation

$$
-\frac{\tan \omega \sqrt{C_{\mathrm{o}} L_{\mathrm{o}}}}{\omega \cdot \sqrt{C_{\mathrm{o}} L_{\mathrm{o}}}}=\frac{C}{C_{\mathrm{o}}}
$$

which has also been given by Guyau. Equation (9) may be solved graphically as above or a table similar to Table I may be prepared giving $\omega \sqrt{C_{0} L_{0}}$ for different values of $\frac{C}{C_{0}}$. More complicated circuits may be solved in a similar manner.

\section{EFFECTIVE RESISTANCE, INDUCTANCE, AND CAPACITY}

In the following the most important practical case of a loading coil in the lead-in and the natural oscillation of lowest frequency alone will be considered. The problem is to replace the antenna
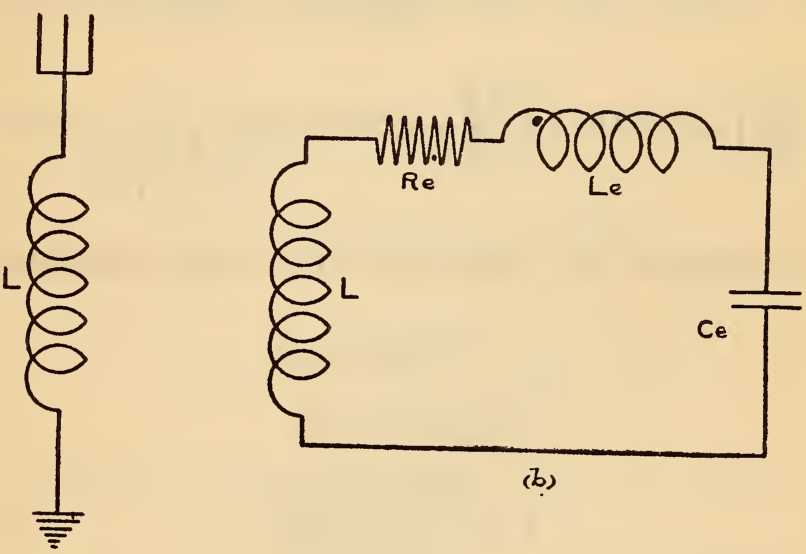

(a)

FIG. 6.-(a) Antenna with loading coil; (b) artificial antenna with lumped constants to represent antenna in $(a)$

of Fig. $6,(a)$, which has a loading coil $L$ in the lead-in and an aerial with distributed characteristics, by a circuit (Fig. 6, (b)) consisting of the inductance $L$ in series with lumped resistance $R_{\mathrm{e}}$, inductance $L_{\mathrm{e}}$, capacity $C_{\mathrm{e}}$, which are equivalent to the aerial. It is necessary, however, to state how these effective values are to be defined.

In practice the quantities which are of importance in an antenna are the resonant wave length or frequency and the current at the current maximum. The quantities $L_{e}$ and $C_{e}$ are therefore defined as those which will give the circuit $(b)$ the same resonant frequency as the antenna in $(a)$. Further the three quantities $L_{\mathrm{e}}, C_{\mathrm{e}}$, and $R_{\mathrm{e}}$ must be such that the current in $(b)$ will be the same 
as the maximum in the antenna for the same applied emf whether undamped or damped with any decrement. These conditions determine $L_{\theta}, C_{e}$, and $R_{\ominus}$ uniquely at any given frequency and are the proper values for an artificial antenna which is to represent an actual antenna at a particular frequency. In the two circuits the corresponding maxima of magnetic energies and electrostatic energies and the dissipation of energy will be the same.

Zenneck ${ }^{4}$ has shown how these effective values of inductance capacity and resistance can be computed when the current and voltage distributions are known. Thus, if at any point $x$ on the oscillator the current $i$ and the voltage $v$ are given by

$$
i=I f(x) ; v=V \phi(x)
$$

where $I$ is the value of the current at the current loop and $V$ the maximum voltage, then the differential equation of the oscillation is

$$
\frac{\partial I}{\partial t} \int R_{1} f(x)^{2} d x+\frac{\partial^{2} I}{\partial t^{2}} \int L_{1} f(x)^{2} d x+\frac{I}{\frac{\left\{f C_{1} \phi(x) d x\right\}^{2}}{\int C_{1} \phi(x)^{2} d x}}=0
$$

where the integrals are taken over the whole oscillator. If we write

$$
\begin{aligned}
& R_{e}=\int R_{1} f(x)^{2} d x \\
& L_{\theta}=\int L_{1} f(x)^{2} d x \\
& C_{\theta}=\frac{\left\{\int C_{1} \phi(x) d x\right\}^{2}}{\int C_{1} \phi(x)^{2} d x}
\end{aligned}
$$

the equation becomes

$$
R_{\ominus} \frac{\partial I}{\partial t}+L_{\mathrm{e}} \frac{\partial^{2} I}{\partial t^{2}}+\frac{I}{C_{\ominus}}=0
$$

which is the differential equation of oscillation of a simple circuit with lumped resistance, inductance, and capacity of values $R_{\mathrm{e}}$, $L_{\mathrm{e}}$, and $C_{\mathrm{e}}$ and in which the current is the same as the maximum in the distributed case. In order to evaluate these quantities, it is necessary only to determine $f(x)$ and $\phi(x)$; that is, the functions which specify the distribution of current and voltage on the oscillator. In this connection it will be assumed that the resistance is not of importance in determining these distributions. 
At the far end of the aerial the current is zero; that is, for $x=l ; i_{l}=0$. From equations (3) and (4) for $x=l$

$$
\begin{aligned}
& v_{l}=\cos \omega t\left(A \cos \omega \sqrt{C_{1} L_{1}} l+B \sin \omega \sqrt{C_{1} L_{1}} l\right), \\
& i_{l}=\sin \omega t \sqrt{\frac{C_{1}}{L_{1}}}\left(A \sin \omega \sqrt{C_{1} L_{1}} l-B \cos \omega \sqrt{C_{1} L_{1}} l\right) ;
\end{aligned}
$$

and since $i_{l}=\mathrm{o}$,

$$
A \sin \omega \sqrt{C_{1} L_{1}} l=B \cos \omega \sqrt{C_{1} L_{1}} l .
$$

From (3), then, we obtain

Hence

$$
v=v_{l} \cos \left(\omega \sqrt{C_{1} L_{1}} l-\omega \sqrt{C_{1} L_{1}} x\right) .
$$

$$
\phi(x)=\cos \left(\omega \sqrt{C_{1} L_{1}} l-\omega \sqrt{C_{1} L_{1}} x\right) .
$$

Now for $x=0$ from (4) we obtain

whence

$$
i_{\mathrm{o}}=-B \sqrt{\frac{C_{1}}{L_{1}}} \sin \omega t=-A \sqrt{\frac{C_{1}}{L_{1}}} \tan \omega \sqrt{C_{1} L_{1}} l \sin \omega t,
$$

and

$$
i=i_{\mathrm{o}} \frac{\sin \left(\omega \sqrt{C_{1} L_{1}} l-\omega \sqrt{C_{1} L_{1}} x\right)}{\sin \omega \sqrt{C_{1} L_{1}} l}
$$

$$
f(x)=\frac{\sin \left(\omega \sqrt{C_{1} L_{1}} l-\omega \sqrt{C_{1} L_{1}} x\right)}{\sin \omega \sqrt{C_{1} L_{1}} l} .
$$

We can now evaluate the expressions (IO), (I I), and (I 2). From (Io)

$$
\begin{aligned}
R_{\mathrm{e}} & =\int_{0}^{l} R_{1} \frac{\sin ^{2}\left(\omega \sqrt{C_{1} L_{1}} l-\omega \sqrt{C_{1} L_{1}} x\right) d x}{\sin ^{2} \omega \sqrt{C_{1} L_{1}} l} \\
& =\frac{R_{1}}{\sin ^{2} \omega \sqrt{C_{1} L_{1}} l}\left[\frac{l}{2}-\frac{\sin 2 \omega \sqrt{C_{1} L_{1}} l}{4 \omega \sqrt{C_{1} L_{1}}}\right] \\
& =\frac{R_{0}}{2}\left[\frac{\mathrm{I}}{\sin ^{2} \omega \sqrt{C_{0} L_{0}}}-\frac{\cot \omega \sqrt{C_{0} L_{0}}}{\omega \sqrt{C_{0} L_{0}}}\right]
\end{aligned}
$$

and from (II) which contains the same form of integral

and from (I2)

$$
L_{\theta}=\frac{L_{0}}{2}\left[\frac{\mathrm{I}}{\sin ^{2} \omega \sqrt{C_{0} L_{0}}}-\frac{\cot \omega \sqrt{C_{0} L_{0}}}{\omega \sqrt{C_{0} L_{0}}}\right]
$$




$$
\begin{gathered}
\text { Bulletin of the Bureau of Standards } \\
C_{\mathrm{e}}=\frac{\left\{\int_{0}^{l} C_{1} \cos \left(\omega \sqrt{C_{1} L_{1}} l-\omega \sqrt{C_{1} L_{1}} x\right) d x\right\}^{2}}{\int_{0}^{l} C_{1} \cos ^{2}\left(\omega \sqrt{\left.C_{1} L_{1} l-\omega \sqrt{C_{1} L_{1}} x\right) d x}\right.} \\
=\frac{C_{1}^{2} \frac{\sin ^{2} \omega \sqrt{C_{1} L_{1} l}}{\left(\omega \sqrt{C_{1} L_{1}}\right)^{2}}}{C_{1}\left(\frac{l}{2}+\frac{\sin 2 \omega \sqrt{C_{1} L_{1}} l}{4 \omega \sqrt{C_{1} L_{1}}}\right)} \\
=C_{0} \frac{1}{\left[\frac{\omega \sqrt{C_{0} L_{0}} \cot \omega \sqrt{C_{0} L_{0}}}{2}+\frac{\omega^{2} C_{0} L_{0}}{2 \sin ^{2} \omega \sqrt{C_{0} L_{0}}}\right]}
\end{gathered}
$$

The expressions (14) and (I5) should lead to the same value for the reactance $X$ of the aerial as obtained before. It is readily shown that

$$
X=\omega L_{\mathrm{e}}-\frac{\mathrm{I}}{\omega C_{\mathrm{e}}}=-\sqrt{\frac{L_{\mathrm{o}}}{C_{\mathrm{o}}}} \cot \omega \sqrt{C_{\mathrm{o}} L_{\mathrm{o}}}
$$

agreeing with equation (6).

It is of interest to investigate the values of these quantities at very low frequencies $(\omega=0)$, frequently called the static values, and those corresponding to the natural frequency of the unloaded antenna or the so-called fundamental of the antenna. Substituting $\omega=0$ in (I3), (I4), and (I5), and evaluating the indeterminant which enters in the first two cases, we obtain for the lowfrequency values

$$
\begin{aligned}
& R_{e}=\frac{R_{\mathrm{o}}}{3} \\
& L_{\mathrm{e}}=\frac{L_{\mathrm{o}}}{3} \\
& C_{\mathrm{e}}=C_{\mathrm{o}}
\end{aligned}
$$

At low frequencies the current is a maximum at the lead-in end of the aerial and falls off linearly to zero at the far end. The effective resistance and inductance are one-third of the values which would obtain if the current were the same throughout. The voltage is, however, the same at all points and hence the effective capacity is the capacity per unit length times the length or $C_{0}$.

At the fundamental of the antenna, the reactance $X$ of equation (6) becomes equal to zero and hence $\omega \sqrt{C_{0} L_{0}}=\frac{\pi}{2}$. Substituting this value in (13), (14), and ( 15 ), 


$$
\begin{aligned}
R_{\mathrm{e}} & =\frac{R_{\mathrm{o}}}{2} \\
L_{\Theta} & =\frac{L_{\mathrm{o}}}{2} \\
C_{\Theta} & =\frac{8}{\pi^{2}} C_{\mathrm{o}} .
\end{aligned}
$$

Hence, in going fron low frequencies up to that of the fundamental of the antenna, the resistance (neglecting radiation and skin effect) and the inductance (neglecting skin effect) increase by 50 per cent, the capacity, however, decreases by about 20 per cent. The incorrect values $\frac{2}{\pi} L_{0}$ and $\frac{2}{\pi} C_{0}$ have been frequently given and commonly used as the values of the effective inductance and capacity of the antenna at its fundamental. These lead also to the incorrect value $L_{\theta}=\frac{L_{0}}{2}$ for the low-frequency inductance. ${ }^{5}$

The values for other frequencies may be obtained by substitution in ( $\left.\mathrm{r}_{3}\right),\left(\mathrm{I}_{4}\right),\left(\mathrm{r}_{5}\right)$. If the value $L$ of the loading coil in the lead-in is given, the quantity $\omega \sqrt{C_{0} L_{0}}$ is directly obtained from Table $\mathrm{r}$.

\section{EQUTVALENT CIRCUIT WITH LUMPED CONSTANTS}

In so far as the frequency or wave length is concerned, the aerial of the antenna may be considered to have constant values of inductance and capacity and the values of frequency or wave length for different loading coils can be computed with slight error using the simple formula applicable to circuits with lumped inductance and capacity. The values of inductance and capacity ascribed to the aerial are the static or low frequency; that is, $\frac{L_{0}}{3}$ for the inductance and $C_{\mathrm{o}}$ for the capacity. The total inductance in case the loading coil has a value $L$ will be $L+\frac{L_{0}}{3}$ and the frequency is given by

$$
f=\frac{\mathrm{I}}{2 \pi \sqrt{\left(L+\frac{L_{0}}{3}\right) C_{0}}}
$$

5 These values are given by J. H. Morecroft in Proc. I. R. E., 5, p. 389; 1917. It may be shown that they lead to correct values for the reactance of the aeria land hence to correct values of frequency, as was verified by the experiments. They are not, however, the values which would be correct for an artificial antenna in which the current must equal the maximum in the actual antenna and in which the energies must also be equal to those in the antenna. The resistance values given by Prof. Morecroft agree with these require ments and with the values obtained here. 
or the wave length in meters by

$$
\lambda=1884 \sqrt{\left(L+\frac{L_{0}}{3}\right) C_{0}}
$$

where the inductance is expressed in microhenrys and the capacity in microfarads. The accuracy with which this formula gives the wave length can be determined by comparison with the exact formula (8). In the second column of Table $I$ are given the values of $\omega \sqrt{C_{0} L_{0}}$ for different values of $\frac{L}{L_{0}}$ as computed by formula (8). Formula (I8) may be written in the form $\omega \sqrt{C_{0} L_{0}}=\frac{I}{\sqrt{\frac{L}{L_{0}}+\frac{1}{3}}}$ so that the values of $\omega \sqrt{C_{0} L_{0}}$, which are proportional to the frequency, may readily be computed from this formula also. These values are given in the third column and the per cent differences in the fourth column of Table $I$. It is seen that formula ( 18 ) gives values for the frequency which are correct to less than I per cent excepting when very close to the fundamental of the antenna; that is, for very small values of $L$. Under these conditions the simple formula leads to values of the frequency which are too high. Hence to the degree of accuracy shown, which is amply sufficient in most practical cases, the aerial can be represented by its static inductance $\frac{L_{0}}{3}$ with its static capacity $C_{0}$ in series, and the frequency of oscillation with a loading coil $L$ in the lead-in can be computed by the ordinary formula applicable to circuits with lumped constants.

In an article by L. Cohen, ${ }^{6}$ which has been copied in several other publications, it was stated that the use of the simple wavelength formula would lead to very large errors when applied to the antenna with distributed constants. The large errors found by Cohen are due to his having used the value $L_{0}$ for the inductance of the aerial, instead of $\frac{L_{\mathrm{o}}}{3}$, in applying the simple formula.

\section{DETERMINATION OF STATIC CAPACITY AND INDUCTANCE}

In applying formula ( 8 ) to calculate the frequency of a loaded antenna, a knowledge of the quantities of $L_{0}$ and $C_{0}$ is required. In applying formula ( 18$), \frac{L_{0}}{3}$ and $C_{0}$ are required. Hence either 
formula may be used if the static capacity and inductance values are known. We will call these values simply the capacity $C_{\mathrm{a}}$ and inductance $L_{\mathrm{a}}$ of the antenna. Hence $C_{a}=C_{0}, L_{\mathrm{a}}=\frac{L_{0}}{3}$ and the wave length from ( 19 ) is given by

$$
\lambda=\mathrm{I} 884 \sqrt{\left(L+L_{\mathrm{a}}\right) C_{\mathrm{a}}}
$$

where inductance is expressed in microhenrys and capacity in microfarads, as before.

The capacity and inductance of the antenna are then readily determined experimentally by the familiar method of inserting, one after the other, two loading coils of known values $L_{1}$ and $L_{2}$ in the lead-in and determining the frequency of oscillation or wave length for each. From the observed wave lengths $\lambda_{1}$ and $\lambda_{2}$ and known values of the inserted inductances, the inductance of the antenna is given by

$$
L_{\mathrm{a}}=\frac{L_{1} \lambda_{2}{ }^{2}-L_{2} \lambda_{1}{ }^{2}}{\lambda_{1}{ }^{2}-\lambda_{2}{ }^{2}}
$$

and the capacity of the antenna from either

$$
\left.\begin{array}{l}
\lambda_{1}=1884 \sqrt{\left(L_{1}+L_{\mathrm{a}}\right) C_{\mathrm{a}}} \\
\lambda_{2}=\mathrm{I} 884 \sqrt{\left(L_{2}+L_{\mathrm{a}}\right) C_{\mathrm{a}}}
\end{array}\right\}
$$

using, preferably, the equation corresponding to the larger valued coil. This assumes that formula (20) holds exactly.

As an example, let us assume that the antenna has $L_{0}=50$ microhenrys and $C_{0}=0.001$ microfarad and that we insert two coils of 50 and 150 microhenrys and determine the wave lengths, experimentally. We know from formula (8) and Table $\mathrm{I}$ that the wave lengths would be found to be $49 \mathrm{r}$ and $77 \mathrm{I} \mathrm{m}$. From the observed wave lengths and known inductances, the value of $L_{a}$ would be found by $(2 \mathrm{I})$ to be

and from (22)

$$
L_{\mathrm{a}}=\mathrm{r} 7.8 \text { microhenrys }
$$

$$
C_{\mathrm{a}}=0.00099_{9} \text { microfarad. }
$$

$C_{a}$ is very close to the assumed value $C_{0}$ but $L_{a}$ differs by 7 per cent from $\frac{L_{0}}{3}$. This accuracy would ordinarily be sufficient. We can, however, by a second approximation, derive from the experimental data a more accurate value of $L_{\mathrm{a}}$. For, the observed value 
of $L_{\mathrm{a}}$ furnishes rough values of $\frac{L_{1}}{L_{\mathrm{o}}}$ and $\frac{L_{2}}{L_{\mathrm{o}}}$, which in this example come out 0.96 and 2.88, respectively. But Table I gives the per cent error of formula (20) for different values of $\frac{L}{L_{0}}$ and shows that this formula gives a 0.7 per cent shorter wave length than $491 \mathrm{~m}$ (or $488 \mathrm{~m}$ ) for $\frac{L}{L_{0}}=0.96$ but no appreciable difference for $\frac{L}{L_{0}}=2.88$. Recomputing $L_{\mathrm{a}}$, using 488 and $77 \mathrm{I} \mathrm{m}$, gives

$$
L_{\mathrm{a}}=0.0168
$$

which is practically identical with the assumed $\frac{L_{0}}{3}$.

6. DETERMINATION OF EFFECTIVE RESISTANCE, INDUCTANCE, AND CAPACITY

When a source of undamped oscillations in a primary circuit induces current in a secondary tuned circuit, the current in the secondary, for a given emf, depends only upon the resistance of the secondary circuit. When damped oscillations are supplied by the source in the primary, the current in the secondary, for a given emf and primary decrement, depends upon the decrement of the secondary; that is, upon the resistance and ratio of capacity to inductance. The higher the decrement of the primary circuit relative to the decrement of the secondary the more strongly does the current in the secondary depend upon its own decrement. This is evident from the expression for the current $I$ in the secondary circuit.

$$
I^{2}=\frac{N E_{0}{ }^{2}}{4 f R^{2} \delta^{\prime}\left(\mathrm{I}+\frac{\delta^{\prime}}{\delta}\right)}
$$

where $\delta^{\prime}$ is the decrement of the primary, $\delta$ that of the secondary, $R$ the resistance of the secondary, $f$ the frequency, $E_{0}$ the maximum value of the emf impressed on the primary, and $N$ the wave-train frequency.

These facts suggest a method of determining the effective resistance, inductance, and capacity of an antenna at a given frequency in which all of the measurements are made at one frequency and which does not require any alteration of the antenna circuit whatsoever. The experimental circuits are arranged as shown in Fig. 7, where $S$ represents a coil in the primary circuit which may be thrown either into the circuit of a source of undamped 
or of damped oscillations. The coil $L$ is the loading coil of the antenna, which may be thrown over to the measuring circuit containing a variable inductance $L^{\prime}$, a variable condenser $C^{\prime}$, and variable resistance $R^{\prime}$. The condenser $C^{\prime}$ should be resistance free and shielded, the shielded terminal being connected to the ground side. First, the undamped source is tuned to the antenna, and then the $L^{\prime} C^{\prime}$ circuit tuned to the source. The resistance $R^{\prime}$ is then varied until the current is the same in the two positions. The resistance of the $L^{\prime} C^{\prime}$ circuit is then equal to $R_{\mathrm{e}}$, the effective resistance of the aerial-ground portion of the antenna and $L^{\prime} C^{\prime}=$ $L_{\mathrm{e}} C_{\mathrm{e}}$. Next, the damped source is tuned to the antenna and the change in current noted when the connection is thrown over to the $L^{\prime} C^{\prime}$ circuit. If the current increases, the value of $C^{\prime}$ is greater than $C_{\mathrm{e}}$, and vice versa. By varying both $L^{\prime}$ and $C^{\prime}$,
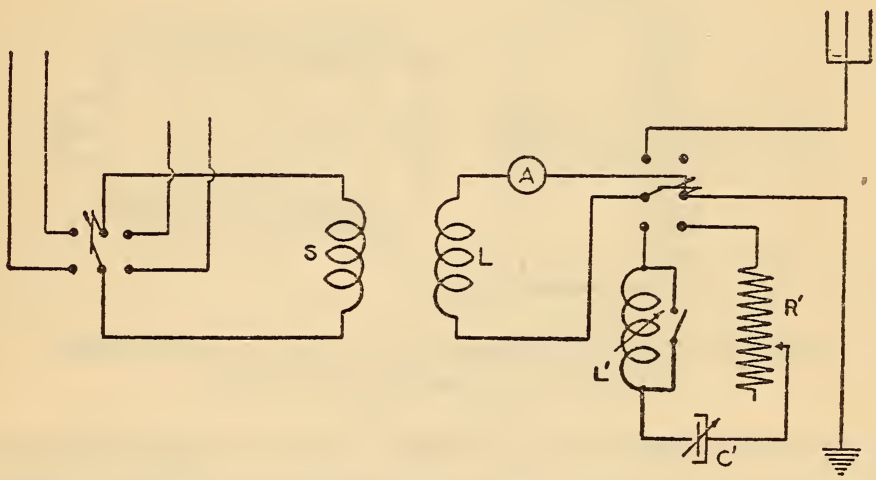

Fig. 7.-Circuits for determining the effective resistance, inductance, and capacity of an antenna

keeping the tuning and $R^{\prime}$ unchanged, the current can be adjusted to the same value in both positions. Then, since $L^{\prime} C^{\prime}=L_{e} C_{e}$ and $\frac{C^{\prime}}{L^{\prime}}=\frac{C_{\mathrm{e}}}{L_{\mathrm{e}}}$, the value of $C^{\prime}$ gives $C_{\mathrm{e}}$ and that of $L^{\prime}$ gives $L_{\mathrm{e}}$. Large changes in the variometer setting may result in appreciable changes in its resistance so that the measurement should be repeated after the approximate values have been found. To eliminate the resistance of the variometer in determining $R_{\mathrm{e}}$, the variometer is shorted and, using undamped oscillations, the resonance current is adjusted to equality in the two positions by varying $R^{\prime}$. Then $R^{\prime}=R_{\mathrm{e}}$. The measurement requires steady sources of feebly damped and strongly damped current. The former is readily obtained by using a vacuum-tube generator. A resonance transformer and magnesium spark gap operating at a low-spark fre$110990^{\circ}-19-14$ 
quency serve very satisfactorily for the latter source, or a single source of which the damping can be varied will suffice. An accuracy of I per cent is not difficult to obtain.

\section{THE INDUCTANCE COIL}

The transmission-line theory can also be applied to the treatment of the effects of distributed capacity in inductance coils. In Fig. 8, $(a)$, is represented a single-layer solenoid connected to a variable condenser $C . A$ and $B$ are the terminals of the coil, $D$ the middle, and the condensers drawn in dotted lines are supposed to represent the capacities between the different parts of the coil. In Fig. 8, (b), the same coil is represented as a line with uniformly distributed inductance and capacity. These
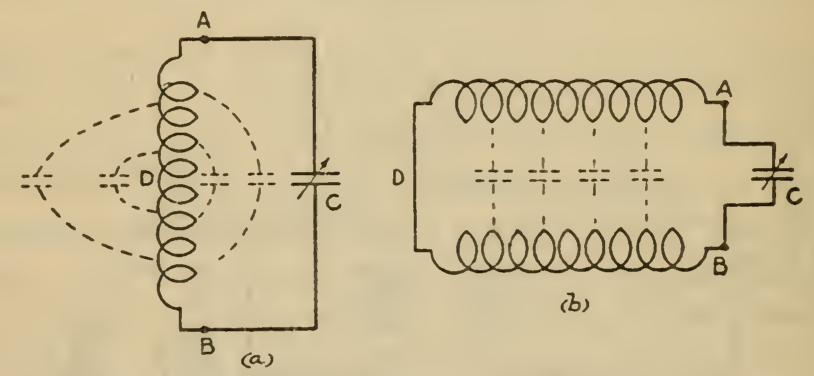

(b)

FIG. 8.-Inductance coil represented as a line with uniform distribution of inductance and capacity

assumptions are admittedly rough but are somewhat justified by the known similarity of the oscillations in long solenoids to those in a simple antenna.

\section{REACTANCE OF THE COIL}

Using the same notation as before, an expression for the reactance of the coil, regarded from the terminals $A B(x=0)$ will be determined considering the line as closed at the far end $D(x=l)$. Equations (3) and (4) will again be applied, taking account of the new terminal condition: that is, for $x=l ; v=0$. Hence

and for $x=0$

$$
A \cos \omega \sqrt{C_{1} L_{1} l}=-B \sin \omega \sqrt{C_{1} L_{1} l}
$$

$$
\begin{aligned}
& v_{0}=A \cos \omega t=-B \tan \omega \sqrt{C_{1} L_{1} l} \cos \omega t \\
& i_{0}=-\sqrt{\frac{C_{1}}{L_{1}} B \sin \omega t}
\end{aligned}
$$


which gives for the reactance of the coil regarded from the terminals $A B$,

or

$$
X^{\prime}=\sqrt{\frac{L_{1}}{C_{1}}} \tan \omega \sqrt{C_{1} L_{1}} l
$$

$$
X^{\prime}=\sqrt{\frac{L_{\mathrm{o}}}{C_{\mathrm{o}}}} \tan \omega \sqrt{C_{\mathrm{o}} L_{\mathrm{o}}}
$$

\section{NATURAL FREQUENCIES OF OSCILLATION}

At low frequencies the reactance of the coil is very small and positive but increases with increasing frequency and becomes infinite when $\omega \sqrt{C_{0} L_{0}}=\frac{\pi}{2}$. This represents the lowest frequency of natural oscillation of the coil when the terminals are open. Above this frequency the reactance is highly negative, approaching zero at the frequency $\omega \sqrt{C_{0} L_{0}}=\pi$. In this range of frequencies the coil behaves as a condenser and would require an inductance across the terminals to form a resonant circuit. At the frequency $\omega \sqrt{C_{0} L_{0}}=\pi$ the coil will oscillate with its terminals short-circuited. As the frequency is still further increased the reactance again becomes increasingly positive.

Condenser Across the Terminals. -The natural frequencies of oscillation of the coil when connected to a condenser $C$ are given by the condition that the total reactance of the circuit shall be zero.

From this we have

$$
X^{\prime}+X_{0}=0
$$

$$
\sqrt{\frac{L_{0}}{C_{0}}} \tan \omega \sqrt{C_{0} L_{0}}=\frac{I}{\omega C}
$$

or

$$
\frac{\cot \omega \sqrt{C_{0} L_{0}}}{\omega \sqrt{C_{0} L_{0}}}=\frac{C}{C_{o}}
$$

This expression is the same as (8) obtained in the case of the loaded antenna, excepting that $\frac{C}{C_{0}}$ occurs on the right-hand side instead of $\frac{L}{L_{0}}$, and shows that the frequency is decreased and wave length increased by increasing the capacity across the coil in a manner entirely similar to the decrease in frequency produced by inserting loading coils in the antenna lead-in.

\section{EQUIVALENT CIRCUIT WITH LUMPED CONSTANTS}

It is of interest to investigate the effective values of inductance and capacity of the coil at very low frequencies. 
Expanding the tangent in equation (23) into a series we find

$$
X^{\prime}=\omega L_{0}\left(I+\frac{\omega^{2} C_{0} L_{0}}{3}+\ldots \ldots\right)
$$

and neglecting higher-power terms this may be written

$$
X^{\prime}=\frac{\left(\omega L_{0}\right)\left(-\frac{3}{\omega C_{0}}\right)}{\omega L_{0}-\frac{3}{\omega C_{0}}}
$$

This is the reactance of an inductance $L_{\circ}$ in parallel with a capacity $\frac{C_{0}}{3}$, which shows that at low frequencies the coil may be regarded as an inductance $L_{0}$ with a capacity $\frac{C_{0}}{3}$ across the terminals and therefore in parallel with the external condenser $C$. Since at low frequencies the current is uniform throughout the whole coil, it is self-evident that its inductance should be $L_{0}$.

Now, the similarity between equations (24) and (8) shows that, just as accurately as in the similar case of the loaded antenna, the frequency of oscillation of a coil with any capacity $C$ across the terminals is given by the formula

$$
f=\frac{I}{2 \pi \sqrt{L_{0}\left(C+\frac{C_{0}}{3}\right)}}
$$

This, however, is also the expression for the frequency of a coil of pure inductance $L_{0}$ with a capacity $\frac{C_{0}}{3}$ across its terminals and which is in parallel with an external capacity $C$. Therefore, in so far as frequency relations are concerned, an inductance coil with distributed capacity is closely equivalent at any frequency to a pure inductance, equal to the low-frequency inductance (neglecting skin effect), with a constant capacity across its terminals. This is a well-known result of experiment, ${ }^{7}$ at least in the case of single-layer solenoids which, considering the changes in current and voltage distribution in the coil with changing frequency, is not otherwise self-evident.

Washington, March 9, i 918.

${ }^{7}$ G. WV. O. Howe, Proc. Phys. Soc. London, 21, D. 251 , 1912; F. A. Kolster, Proc. Inst. Radio Eng., 1, p. 19, ז913; J. C. Hubbard, Phys. Rev., 9, p. 529, 1917. 